\title{
Concurrent Pulmonary, Intracranial, Intramedullary Tuberculoma, and Their Response to Conservative Management
}

\author{
${ }^{1}$ Division of Spine, Department of Orthopaedics, JN Medical \\ College, Wardha, Maharashtra, India \\ 2Department of Orthopaedics, JN Medical College, Wardha, \\ Maharashtra, India \\ ${ }^{3}$ Department of Radiodiagnosis, JN Medical College, Wardha, \\ Maharashtra, India
}

Sohael Khan ${ }^{1}$ Suvarn Gupta ${ }^{2}$ Shashank Jain² Shraddha Singhania ${ }^{3}$

\begin{abstract}
Address for correspondence Shraddha Singhania, MBBS, DMRD, Department of Radiodiagnosis, JN Medical College, Wardha 442005, Maharashtra, India (e-mail: shraddha2527@gmail.com).
\end{abstract}

Int J Recent Surg Med Sci 2018;4:88-90

\begin{abstract}
Keywords

- intraspinal tuberculoma

- intramedullary tuberculoma

- tuberculoma

Tuberculomas of the central nervous system are common. However, the incidence of intraspinal intramedullary tuberculomas is very low, and a combination of intramedullary, intracranial, and pulmonary tuberculomas is extremely rare. We report a case of tuberculoma involving the brain and spine with pulmonary tuberculosis in a 46-year-old man initially presenting with back pain and weakness of both lower extremities. Conservative treatment with antituberculous medications resulted in complete resolution of his symptoms.
\end{abstract}

\section{Introduction}

Tuberculosis, even after the awareness and availability of variety of treatments, is the most commonly encountered problem of developing countries. Intramedullary tuberculomas are rare and constitute only 0.2 to $5 \%$ of all tuberculomas of the central nervous system (CNS). ${ }^{1}$ The combination of intramedullary and intracranial tuberculomas is extremely rare, and only five cases have been reported in the literature so far. ${ }^{2}$

Tuberculomas are tumor-like masses resulting from enlargement or coalescence of caseated tubercles. CNS tuberculomas most commonly affect the intracranial compartment; spinal intramedullary tuberculomas (SITs) are very rare. ${ }^{3}$ Concurrent intracranial and intramedullary tuberculomas are extremely uncommon and mostly described in immune-compromised patients. ${ }^{2,4}$ SITs commonly affect the thoracic spinal cord, with the cervical segment being an unusual site of such occurrence. ${ }^{5}$

Here, we present a case of a man who came with the complaint of lower limb paralysis and bowel and bladder incontinence and was accidentally diagnosed with intraspinal tuberculoma. There was also a coexistent cerebellar tuberculoma with tubercular pleural effusion.

\section{Case Report}

A 46-year-old man was admitted with back pain and motor weakness of both lower extremities for 2 weeks. The patient gradually developed lower limb paralysis with bowel and bladder incontinence. Magnetic resonance imaging (MRI) of the lumbar spine revealed degenerative changes; however, screening of the whole spine (T2WI sagittal view) showed a well-defined rounded hyperintense lesion at D9 vertebral body level and in the cerebellar vermis. Complete evaluation of the dorsal spine and brain was done, which revealed hyperintense lesions at D9 vertebral body level and cerebellar vermis on T2-weighted images causing cord expansion. The lesion showed prominent ring enhancement with contrast medium (-Figs. 1, 2). The patient showed negative for human immunodeficiency virus (HIV), but his chest X-ray revealed increased bronchovascular markings and right-sided pleural effusion ( - Fig. 3 ), which on diagnostic tapping came out positive for tuberculosis. Ultrasound of the abdomen was done to rule out abdominal Koch's. He was treated with rifampicin $600 \mathrm{mg}$, isoniazid $300 \mathrm{mg}$, pyrazinamide $1,500 \mathrm{mg}$, ethambutol $1,200 \mathrm{mg}$, and pyridoxine $25 \mathrm{mg}$. Within 1 week, he started showing signs of recovery in the form of gradual improvement in his
License terms

()(1) $\Theta \circledast$
DOI https://doi.org/ ISSN 2455-7420. 




Fig. $1 \mathrm{MRI}$ of the spine saggital section shows well-defined ring-enhancing lesion at D9 vertebral body level. MRI, magnetic resonance imaging.

power. At discharge at the end of 2 weeks, he was able to walk with the aid of a walker. He continued making steady improvement in his neurologic status. An MRI done at the end of 3 months showed a significant decrease in the size of the cerebral and cerebellar tuberculomas with resolution of edema around the intramedullary tuberculoma. At the end of 1 year, he demonstrated a full neurologic recovery, and a repeat MRI showed complete resolution of the cerebral and cerebellar tuberculomas. However, gliosis was seen in the dorsal segments of the spinal cord. Chest X-ray came out to be normal.

\section{Discussion}

Tuberculosis is very common in the developing countries such as ours; however, intramedullary spinal tuberculomas are

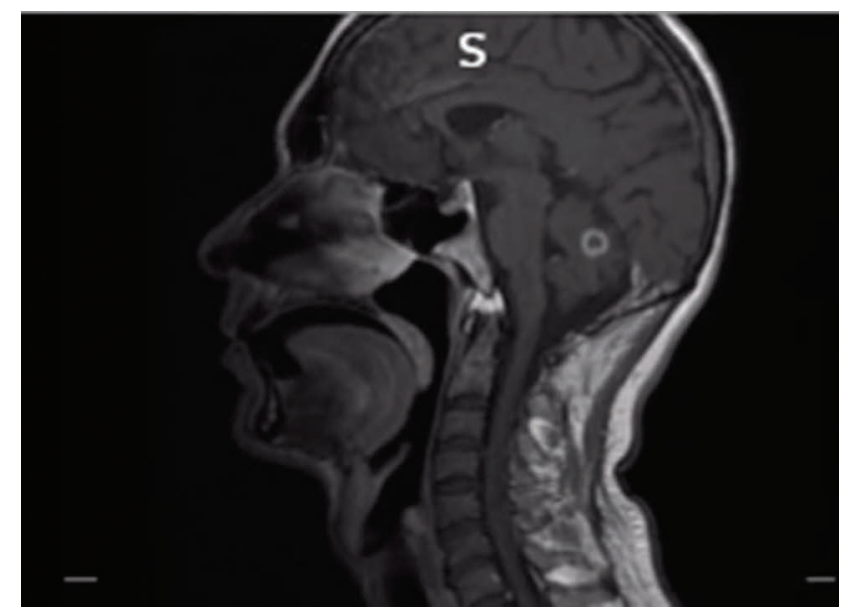

Fig. $2 \mathrm{MRI}$ of the brain saggital section shows well-defined ring-enhancing lesion in the cerebellar vermis. MRI, magnetic resonance imaging.

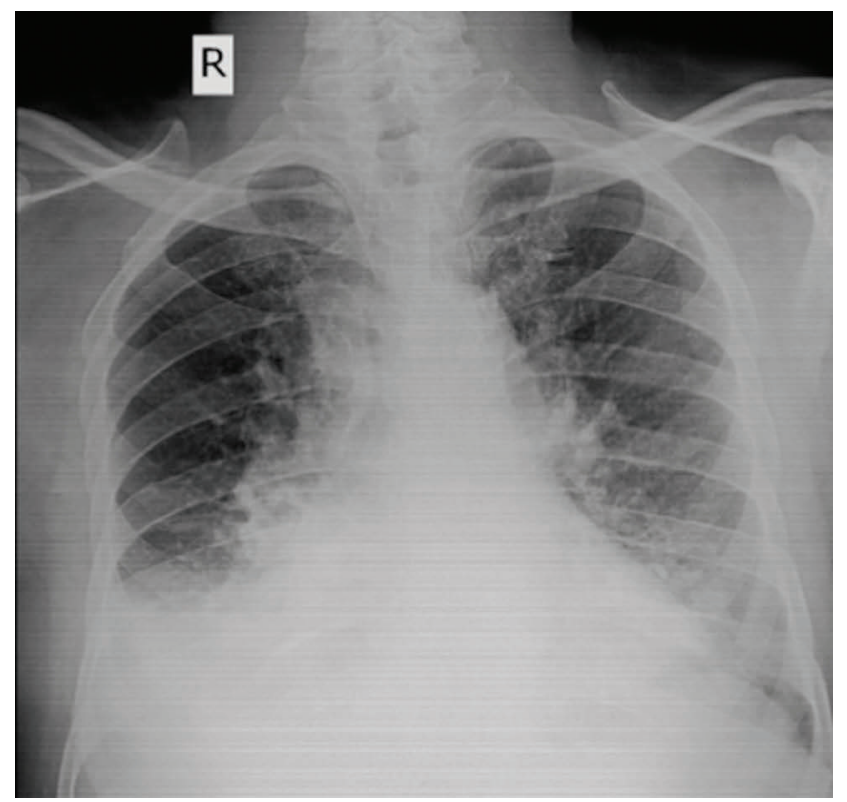

Fig. 3 PA view of chest $X$-ray reveals right-sided pleural effusion with increased bronchovascular markings. PA, postero-anterior.

very rare and concurrent appearance of spinal, intracerebellar, and pulmonary tuberculosis is rarer.

SITs can occur within the cord primarily or commonly occurs secondary to an existing focus elsewhere in the body and spreads through hematogenous route or less likely by the cerebrospinal fluid like in intraspinal tuberculoma. Our patient had cerebellar vermian tuberculoma in addition to the dorsal intramedullary lesion. Such concurrent lesions occur more likely in immune-compromised patients. However, our patient was negative for HIV. Because the patient came with lower limb paralysis and bowel and bladder incontinence, prophylactically screening of the whole spine with sagittal screening of the brain was done. Park and Song ${ }^{6}$ have recommended prophylactic cranial imaging in patients with intramedullary spinal cord tuberculoma, so that intracranial tuberculomas can be ruled 
out. Thacker and Puri ${ }^{2}$ reported a case of a young girl who presented with progressive paraparesis, and imaging revealed intramedullary tuberculoma with incidental finding of multiple intracranial tuberculomas. Yen et $\mathrm{al}^{7}$ reported a case of 67-year-old man with known pulmonary tuberculosis who developed compressive myelopathy of the spine. Imaging revealed an intramedullary spinal tuberculoma with multiple intracranial tuberculomas. In both the cases, the intracranial lesions were incidentally discovered, as in our case, all the tuberculomas were discovered incidentally and chest X-ray also showed tuberculous fluid, even when the patient did not give any chest complaints.

Tuberculomas are best detected in MRI, and MRI also eliminates the need for invasive procedures., ${ }^{8,9}$ Tuberculomas are hypoisointense lesions on T2-weighted images (T1WI) and low-intensity lesions with or without central hyperintensity (due to varying amount of caseous necrosis) on T2-weighted images (T2WI). Intense ring enhancement is seen post-contrast. The differential diagnosess of intramedullary tuberculomas are neurocysticercosis, metastasis, etc. However, the resolution of the lesions after antituberculous treatment confirmed our diagnosis.

The best treatment of intramedullary tuberculoma is still a topic of debate. Both surgical and medical treatments have given good results in different case. ${ }^{6,10}$ However, some authors recommend medical treatment of intramedullary tuberculomas as they give good results. ${ }^{2}$ The chief complaint of our patient was paralysis of both lower extremities, with urinary incontinence. The patient's neurologic symptoms improved post-antituberculous treatment.

This case was reported to emphasize on the fact that intramedullary tuberculoma can be associated with concurrent presence of intracranial tuberculomas and pulmonary Koch's that could be asymptomatic. Although screening of the brain led us to the diagnosis of intracranial tuberculoma, there is no such evidence recommending such screening in routine cases, but once tuberculoma is detected, other common areas such as the chest and abdomen should be investigated. Our case also stands by the studies that recommend medical treatment and state that conservative treatment can achieve a good clinical outcome and that surgical intervention may not always be indicated. Surgery should be reserved for cases showing progressive deficit in spite of adequate medical management.

\section{Conflict of Interest}

None.

\section{References}

1 Torii H, Takahashi T, Shimizu H, Watanabe M, Tominaga T. Intramedullary spinal tuberculoma-case report. Neurol Med Chir (Tokyo) 2004;44(5):266-268

2 Thacker MM, Puri AI. Concurrent intra-medullary and intra-cranial tuberculomas. J Postgrad Med 2004;50(2): 107-109

3 MacDonnell AH, Baird RW, Bronze MS. Intramedullary tuberculomas of the spinal cord: case report and review. Rev Infect Dis 1990;12(3):432-439

4 Muthukumar N, Venkatesh G, Senthilbabu S, Rajbaskar R. Surgery for intramedullary tuberculoma of the spinal cord: report of 2 cases. Surg Neurol 2006;66(1):69-74, discussion 74

5 Kayaoglu CR, Tuzun Y, Boga Z, Erdogan F, Gorguner M, Aydin IH. Intramedullary spinal tuberculoma: a case report. Spine 2000;25(17):2265-2268

6 Park HS, Song YJ. Multiple tuberculoma involving the brain and spinal cord in a patient with miliary pulmonary tuberculosis. J Korean Neurosurg Soc 2008;44(1):36-39

7 Yen HL, Lee RJ, Lin JW, Chen HJ. Multiple tuberculomas in the brain and spinal cord: a case report. Spine 2003; 28(23):E499-E502

8 Lin J, Feng $\mathrm{H}$, Ai S, Wang X. Intramedullary cervical tuberculoma. Spinal Cord 2006;44(12):809-812

9 Shenoy SN, Raja A. Concurrent intramedullary and intracerebral tuberculomas. Neurol India 2004;52(4):514-516

10 Ju HB, Guo DM, Chen FF. Intramedullary cervical tuberculoma: a case report with note on surgical management. Int Surg 2015;100(1):133-136 